\title{
Hidrocolpos e hidrometrocolpos neoanatal: a propósito de un caso clínico
}

\author{
CAROLINA GARFIAS VON F. ${ }^{1}$, ANTONIO BARRERA N. ${ }^{2}$, \\ RICARDO ZUBIETA A. ${ }^{3}$, FRANCISCA UGARTE P. ${ }^{2,4}$ \\ 1. Médico Pediatra, Unidad de Endocrinología, Hospital Dr. Exequiel González Cortés. \\ 2. Endocrinólogo Infantil, Unidad Endocrinología Hospital Dr. Exequiel González Cortés. \\ 3. Urólogo Infantil, Servicio de Cirugía, Hospital Exequiel González Cortés. \\ 4. Departamento de Pediatría, Universidad de Los Andes.
}

\begin{abstract}
Neonatal hydrocolpos and hydrometrocolpos: a clinical case

Hydrocolpos and hydrometrocolpos is a condition characterized by a cystic dilatation od the vagina and/or uterus with accumulation of fluid as a result of congenital vaginal obstructions. It can be of secretory or urinary types, the last one when a vagino-vesical communication develops such as a sinus or chloaca. Other causes are vaginal septum, imperforated hymen. Clinical Case: Ten day old newborn, 46 XX with genital virilization (Prader IV) confirmed as due to Congenital Adrenal Hyperplasia, sonogram showed dilated vagina with fluid content due to neonatal hydrocolpos. Conclusion: It is important to maintain a high index of suspicion when a female newborn shows urogenital sinus, chloaca, genital virilization or imperforated hymen, as well as a female newborn with an abdominal mass. Diagnostic test of choice is a sonogram. Evaluation must be completed by a multidisciplinary team, including urology, endocrinology and pediatric gynecology for optimal patient management.
\end{abstract}

(Key words: Hidrocolpos, hidrometrocolpos, hiperplasia suprarrenal congénita).

Rev Chil Pediatr 2011; 82 (2): 137-141

\section{RESUMEN}

El Hidrocolpos e Hidrometrocolpos es una condición caracterizada por dilatación quística de la vagina y/o del útero, con acumulación de líquido como resultado de obstrucciones vaginales congénitas. Puede ser de tipo secretorio o urinario, este último ocurre cuando existe comunicación vagino-vesical, como en el seno urogenital o anomalía tipo cloaca. Otras causas son septo vaginal, himen imperforado, malformación tipo cloaca y senourogenital. Caso: Recién nacida de 10 días, 46 XX, con virilización de genitales grado IV de Prader, cuyo estudio confirmó una Hiperplasia Suprarrenal Congénita y cuya ecografía demostró una vagina dilatada con contenido liquido correspondiendo a un hidrocolpos neonatal.

(Palabras clave: Hidrocolpos, hidrometrocolpos, hiperplasia suprarrenal congénita).

Rev Chil Pediatr 2011; 82 (2): 137-141

Trabajo recibido el 09 de marzo de 2011, devuelto para corregir el 19 de abril de 2011, segunda versión el 24 de abril de 2011, aceptado para publicación el 29 de abril de 2011.

Correspondencia a:

Dra. Carolina Garfias

E-mail: cpgarfias@gmail.com 


\section{Introducción}

El hidrocolpos e hidrometrocolpos es una dilatación quística de la vagina o de el útero y vagina, respectivamente, con acumulación de líquido como resultado de obstrucciones vaginales congénitas ${ }^{1}$. Se manifiesta durante el periodo neonatal, la adolescencia o en la adultez. ${ }^{1}$ Puede ser de tipo secretorio o urinario, este ultimo ocurre cuando existe comunicación vagino-vesical, como en el seno urogenital o anomalía tipo cloaca ${ }^{2}$. La etiopatogenia puede ser variada durante el desarrollo embrionario: falla en la canalización de la placa vaginal a distintos niveles (ej. septo vaginal), interrupción de la normal canalización del intestino posterior (ej. cloaca y el seno urogenital) y como consecuencia de virilización de genitales externos en un recién nacido femenino con Hiperplasia suprarrenal congénita (HSRC).

\section{Caso Clínico}

Se presenta el caso clínico de un recién nacido de 38 semanas producto de un parto eutócico, que presentó liquido amniótico con meconio, apgar 7-9 y síndrome de distress respiratorio leve y que peso al nacer $3480 \mathrm{~g}$ y midió $49 \mathrm{~cm}$. Con antecedentes de padres sanos, consanguíneos (primos hermanos) y un hermano sano.

Al examen físico del recién nacido destacó alteración de genitales con falo de $2 \mathrm{~cm}$ de longitud y 0,6 cm de ancho, meato urinario en extremo anterior de rafe perineal, zona labioescrotal con fusión completa e hiperpigmentada, sin gónadas palpables en su interior. (Figura 1 a y $1 b)$.

Se solicitaron exámenes de laboratorio y de imágenes como parte del estudio de genitales ambiguos. Dentro de los exámenes de laboratorio destacaba cariograma $46 \mathrm{XX}, 17$ hidroxi progesterona de $69 \mathrm{ng} / \mathrm{ml}(\mathrm{VR}<10 \mathrm{ng} / \mathrm{ml})^{2}$ y actividad de renina plasmática (ARP) 11,5 ng/ $\mathrm{ml} / \mathrm{hr}$ (VR: 7,3 \pm 3,39); hiponatremia leve, hiperkalemia leve y glicemias normales.

Se realizó ecografía pelviana transabdominal a los 5 días de vida que mostró moderado aumento de tamaño de glándulas suprarrenales con ecoestructura conservada, glándula suprarrenal derecha 4 x 0,9 cm, glándula suprarrenal Izquierda de 3,4 x 0,9 cm (el tamaño normal de las glándulas suprarrenales en el periodo neonatal es de largo 0,9 a 3,6 cm con una media de $1,5 \mathrm{~cm}$ y ancho 0,2 a $0,5 \mathrm{~cm}$ con una media de $0,3 \mathrm{~cm})^{3}$ (figuras 2 y 3). En la pelvis se observó una estructura líquida ovalada de contornos parcialmente delimitados en situación vesicorectal de 6,1 cm de eje longitudinal con 2,4 cm de grosor x $3 \mathrm{~cm}$ de ancho, en cuyo extremo proximal se observa una estructura sólida que puede corresponder a útero asociado a un hidrocolpos. No se visualizan gónadas (figuras 4 y 5).

Se diagnosticó Desorden de la Diferenciación Sexual (DSD) XX, Hiperplasia suprarrenal congénita perdedora de sal con virilización grado IV de Prader e Hidrocolpos neonatal.
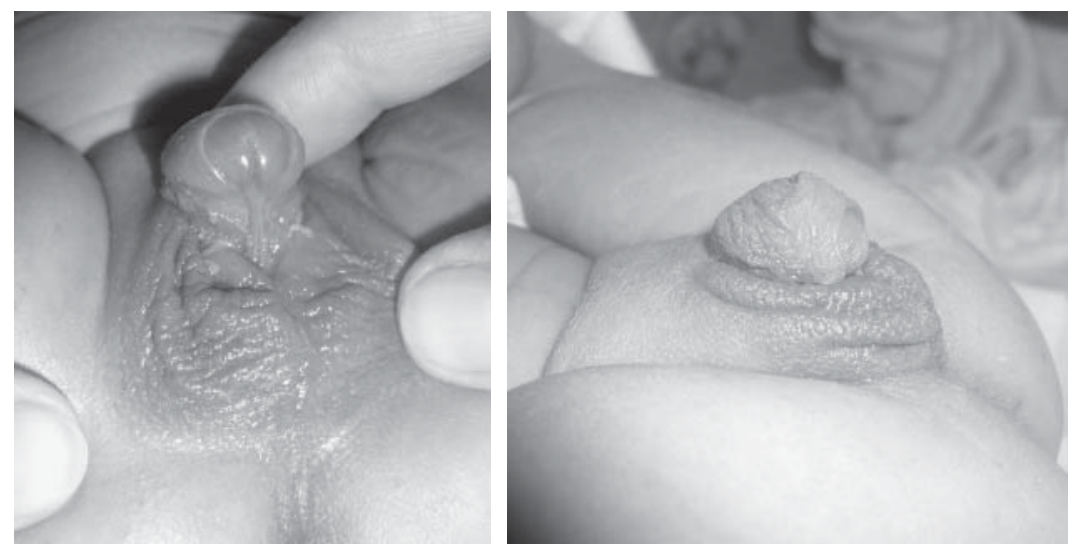

Figura 1a y 1b. Falo de 1 x 0,6 $\mathrm{cm}$ con meato urinario en parte anterior del rafe y fusión completa de rafe labioescrotal. 


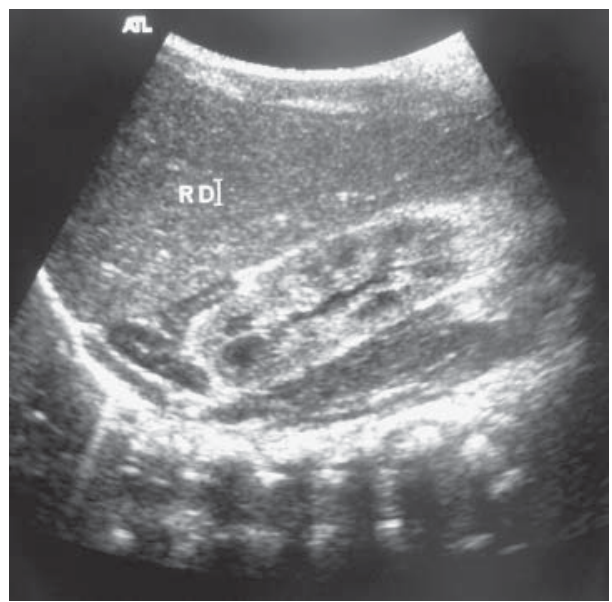

Figura 2. Aumento de tamaño de glándulas suprarrenales con ecoestructura conservada. Gándula suprarrenal derecha $4 \times 0,9 \mathrm{~cm}$.

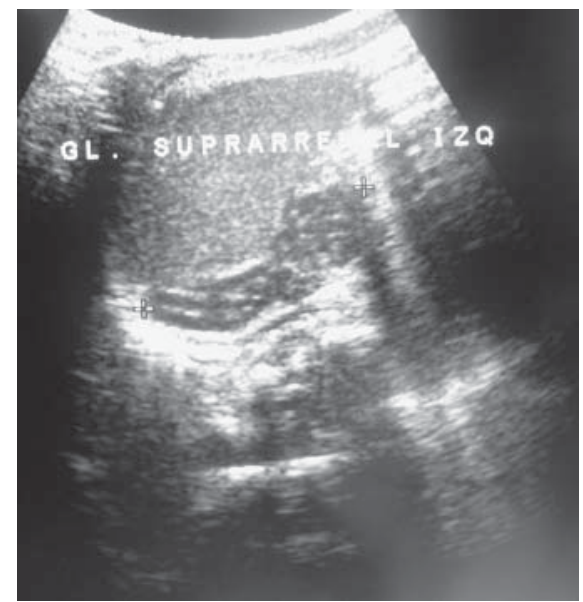

Figura 3. Aumento de tamaño de glándulas suprarrenales con ecoestructura conservada. Glándula suprarrenal Izquierda de 3,4 x 0,9 cm.
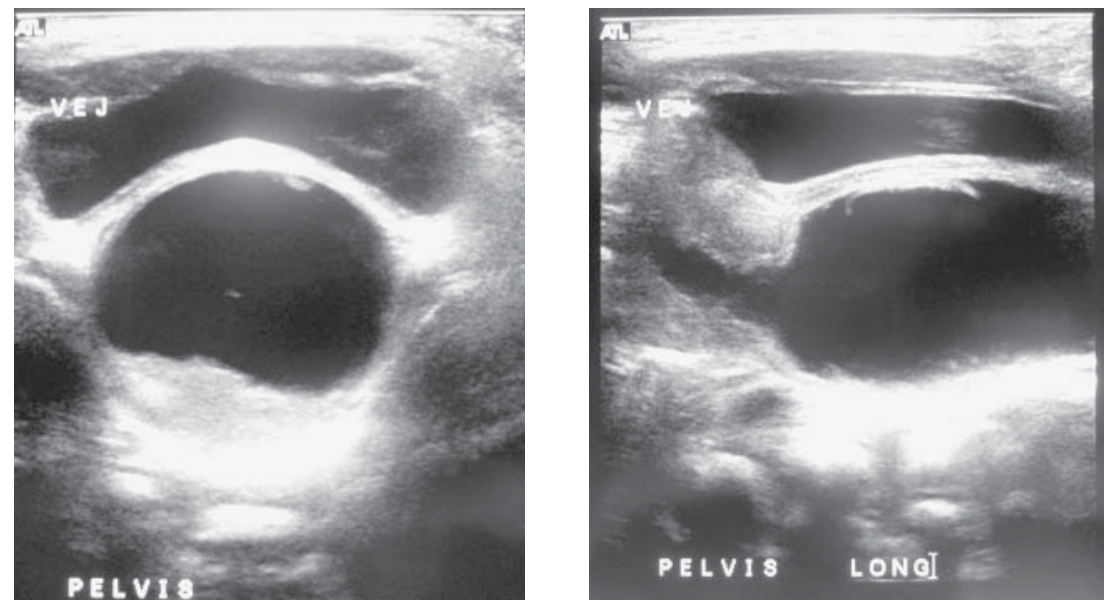

Figura 4 y 5. En la pelvis se observa estructura liquida ovalada, de contornos parcialmente delimitados en situación vesico-rectal de 6,1 cm de eje longitudinal con 2,4 cm de grosor $\mathrm{x} 3 \mathrm{~cm}$ de ancho, en cuyo extremo proximal se observa una estructura sólida que puede corresponder a útero asociado a un hidrocolpos. No se visualizan gónadas.

Se inició tratamiento a los 10 días de vida con Hidrocortisona 2,5 mg cada 12 horas vo $\left(20 \mathrm{mg} / \mathrm{m}^{2} /\right.$ día) y Florinef (Fluohidrocortisona) 0,05mg cada 24 horas vo. Evolucionó en buenas condiciones generales, con buen incremento pondoestatural y fue dad de alta a los 20 días de vida. Se controló $17 \mathrm{OH}$ progesterona al mes de vida con resultado de 7,2 ng/ml (vn $<7,5 \mathrm{ng} / \mathrm{ml}$ ).

Una ecografía pelvica de control a los 23 días de vida, demostró significativa disminu- ción de las glándulas suprarrenales y persiste de imagen de dilatación vaginal (hidrocolpos) de 22 cc de volumen, de aspecto homogéneo.

\section{Discusión}

En el período neonatal el hidrocolpos e hidrometrocolpos se pueden manifestar como masa abdominal, masa que protruye por vía vaginal, sepsis, dificultad respiratoria, infec- 
ción del tracto urinario o ser un hallazgo ecográfico, como el caso de nuestra paciente.

Esta condición puede permanecer silente durante la infancia hasta la adolescencia, donde se presenta como dolor abdominal, dolor lumbar, alteraciones miccionales, amenorrea y hematocolpos o hematometra (colección de producto de la menstruación en vagina o útero y vagina). En la mujer adulta se presenta con dispareunia, dolor lumbar, infertilidad ${ }^{1}$.

Los niveles elevados de estrógenos durante el periodo neonatal aumentan la secreción de las glándulas de la mucosa cervical, favoreciendo la acumulación de fluido seromucoso, razón por la cual esta patología se hace sintomática favoreciendo el diagnóstico ${ }^{4}$. Durante la infancia la no estrogenización de los tejidos hace que esta condición pueda permanecer silente hasta la adolescencia, en donde la menarquia produce hidrohematocolpos.

Las causas de hidrocolpos e hidrometrocolpos son la mayor parte de las veces esporádicas como himen inperforado, septum vaginal tranverso, senourogenital y malformación cloacal $^{1}$.

Se han descrito dos condiciones genéticas de transmisión autosómicas recesivas que presentan esta condición, como el sindrome de McKusick Kaufman caracterizado por atresia vaginal con hidrometrocolpos, polidactilia post axial y cardiopatias congénitas y el síndrome de Bardet-Bield caracterizado por retardo mental, retinopatía pigmentaria, hipogonadismo, obesidad, en niñas puede existir atresia vaginal con hidrometrocolpos ${ }^{5}$.

Los casos descritos de hidrocolpos o hidrometrocolpos son escasos en la literatura, con publicación de casos aislados y sin existir datos de incidencia. Sarmiento y cols describieron el caso de una recién nacida que presentaba una tumoración renitente que protruía a través del introito vaginal al llanto, la ecografía demostró una imagen ecolúcida en hipogastrio de 4,3 $\mathrm{x} 3,1 \mathrm{~cm}$, con liquido en su interior, la vejiga se visualizaba llena de contornos regulares e hidroureteronefrosis izquierda, por efecto de masa. Riñón derecho normal. Se diagnóstico hidrocolpos secundario a himen imperforado, se realizó himenotomia al cuarto día de vida con buena evolución ${ }^{6}$.
El hidrocolpos e hidrometrocolpos en el periodo neoanatal puede dar síntomas por compresión y efecto de masa, se han descrito hidroureteronefrosis, alteraciones defecación por obstrucción rectal, edema de extremidades inferiores por compresión de la vena cava inferior. ${ }^{4}$ En nuestra paciente no se evidenció en la ecografía compromiso obstructivo renal. Durante el examen físico presentó evacuación rectal normal y no se observó edema de extremidades inferiores.

El diagnóstico diferencial debe realizarse en el periodo neonatal o prenatal con duplicación vesical, quiste ovárico o quiste meconial. En la literatura se han descrito casos de hidrocolpos de diagnóstico prenatal. ${ }^{4,7}$ Subramanian et al, describieron el caso de una paciente de 26 años con 36 semanas de gestación que durante la ecografía fetal se observa una masa grande abdomino pélvica separada de la vejiga, se realiza RMN fetal que muestra una estructura dilatada tubular pélvica posterior a la vejiga y con extensión al piso pélvico que correspondía vagina y útero dilatados en un feto femenino. Después del nacimiento se comprobó una recién nacida de sexo femenino con senourogenital $^{2}$. La RMN es el examen de elección para el estudio de estructuras abdomino pélvicas en el periodo prenatal ${ }^{7,8}$.

Una caso bastante similar al de nuestra paciente fue descrito por Couper et al, que reportaron el caso de una recién nacida con HSRC, al examen físico presentaba clitoromegalia con fusión labial completa y un orificio uretral sin apertura vaginal. A las 5 semanas de vida consulta por masa palpable abdominal, la ecografía abdominal demuestra vagina marcadamente dilatada, diagnosticándose hidrocolpos ${ }^{9}$.

Dentro de las complicaciones descritas en el período neonatal se encuentran, distress respiratorio, compresión de vías urinarias, en adolescentes y mujeres adultas puede producirse infección del contenido con piometra o piocolpos.

En resumen, consideramos importante tener un alto índice de sospecha frente a un recién nacido de sexo femenino con seno urogenital o alteración tipo cloaca, virilización de genitales o himen imperforado, así como frente a una recién nacida con una masa abdominal. El 
examen de elección para el diagnóstico es la ecografía. La evaluación debe realizarse por equipo multidisciplinario con urología, endocrinología y ginecología infantil. El tiempo de la cirugía dependerá de cada caso y del riesgo de infección (piometra o piocolpos) que exista.

\section{Referencias}

1.- $\quad$ Nazir Z, Rizzi R, Qureshi R, Khan ZS, Khan Z: Congenital vaginal obstructions:varied presentation and outcome. Pediatr Surg Int 2006; 22: 749-53.

2.- Soriano L, Velásquez: Hiperplasia Suprarrenal Congénita. Pediatr Integral 2007; 11: 7: 601-10.

3.- Pediatric Radiology Normal Measurements. http://www. ohsu.edu/radiology/teach/kojima/adrenal.htm

4.- Subramanian S, Raju S, Gamanagatti S, Agarwala S, Gupta P, Kumar S: Antenatal MR diagnosis of urinary htdrometrocolpos due to urogenital sinus. Pediatric Radiol 2006; 36: 1086-9.

5.- Punnachalil M, Al Sanna N, Ayyatb F: Hydrometrocolpos and acute renal failure: A rare neonatal presentation of Bardet-Biedl syndrome. J Pediatr Urol 2008; 4 (4): 313-6.

6.- Sarmiento Y, Crespo A, Sánchez I, Méndez YR: Hidrocolpos neonatal. Rev Cub Pediatr 2009; 81 (4): 93-7.

7.- Nussbaum AR, Sanders RC, Gearhart JP: Obstructed uterovaginal anomalies: demostration with sonography. Radiology 1991; 179:79-83.

8.- Hayashi S, Sago H, Kashima K, Kitano K; Kuroda T; Honna T: Prenatal diagnosis of fetal hydrometrocolpos secondary to a cloacal anomaly by magnetic resonante Imaging. Ultrasound Obstet Gynecol 2005; 26: 577-579.

9.- Couper J, Hut son JM, Warne GL: Hydrometrocolpos following prenatal daxamethasone treatment for congenital adrenal hiperplasia (21-hydroxylase deficiency). Eur J Pediatr 1993; 152: 9-11. 